\title{
Ventral Tegmental Area/Midbrain Functional Connectivity and Response to Antipsychotic Medication in Schizophrenia
}

\author{
Jennifer A Hadley', Rodolphe Nenert ${ }^{2}$, Nina V Kraguljac', Mark S Bolding ${ }^{3}$, David M White', \\ Frank M Skidmore ${ }^{4}$, Kristina M Visscher ${ }^{2}$ and Adrienne C Lahti*, I \\ 'Department of Psychiatry and Behavioral Neurobiology, University of Alabama at Birmingham, Birmingham, AL, USA; ${ }^{2}$ Department of \\ Neurobiology, University of Alabama at Birmingham, Birmingham, AL, USA; ${ }^{3}$ Department of Radiology, University of Alabama at Birmingham, \\ Birmingham, AL, USA; ${ }^{4}$ Department of Neurology, University of Alabama at Birmingham, Birmingham, AL, USA
}

\begin{abstract}
Medication management in schizophrenia is a lengthy process, as the lack of clinical response can only be confirmed after at least 4 weeks of antipsychotic treatment at a therapeutic dose. Thus, there is a clear need for the discovery of biomarkers that have the potential to accelerate the management of treatment. Using resting-state functional MRI, we examined the functional connectivity of the ventral tegmental area (VTA), the origin of the mesocorticolimbic dopamine projections, in 21 healthy controls and 21 unmedicated patients with schizophrenia at baseline (pre-treatment) and after I week of treatment with the antipsychotic drug risperidone (I-week posttreatment). Group-level functional connectivity maps were obtained and group differences in connectivity were assessed on the groups' participant-level functional connectivity maps. We also examined the relationship between pre-treatment/I-week post-treatment functional connectivity and treatment response. Compared with controls, patients exhibited significantly reduced pre-treatment VTA/midbrain connectivity to multiple cortical and subcortical regions, including the dorsal anterior cingulate cortex (dACC) and thalamus. After I week of treatment, VTA/midbrain connectivity to bilateral regions of the thalamus was re-established. Pre-treatment VTA/midbrain connectivity strength to dACC was positively correlated with good response to a 6-week course of risperidone, whereas pre-treatment VTA/midbrain connectivity strength to the default mode network was negatively correlated. Our findings suggest that VTA/midbrain resting-state connectivity may be a useful biomarker for the prediction of treatment response.

Neuropsychopharmacology (2014) 39, 1020-1030; doi:I0.1038/npp.2013.305; published online 4 December 2013
\end{abstract}

Keywords: resting-state functional connectivity; default mode network; anterior cingulate cortex; treatment response; ventral tegmental area; schizophrenia

\section{INTRODUCTION}

Antipsychotic medications are the only effective treatment for positive symptoms in schizophrenia, but have little effect on negative or cognitive symptoms. All antipsychotics act on the dopamine $\mathrm{D}_{2}$ receptor with similar efficacy, but clinical response is variable and currently unpredictable. Approximately $30 \%$ of patients will not improve with medications (Lieberman et al, 2005), and 60\% will show sub-optimal response (Harrow et al, 1997). Each year, onefourth of all patients are switched to a different antipsychotic in an effort to better manage their illness (Lieberman et al, 2005). An adequate medication trial requires at least 4 weeks of treatment at a therapeutic dose before lack of clinical response can be confirmed (Marder

*Correspondence: Dr AC Lahti, Department of Psychiatry and Behavioral Neurobiology, University of Alabama at Birmingham, SC 50I, I 530 3rd Ave South, Birmingham, AL 35294-00 I7, USA, Tel: +205996 6776, Fax: +205975 4879, E-mail: alahti@uab.edu Received 12 June 2013; revised 18 October 2013; accepted 21 October 2013; accepted article preview online 29 October 2013 et al, 2002), despite data indicating the presence of drug effects after as early as 1 week (Agid et al, 2003). Therefore, there is a clear clinical need for a biomarker to assist with the determination of drug effectiveness early in the course of treatment, or before medication is initiated.

One promising neuroimaging technique to evaluate antipsychotic response is functional connectivity (FC), which measures the temporal coherence of spontaneous neural activity between distinct brain regions (Biswal et al, 1995; Damoiseaux et al, 2006), allowing for the identification of large-scale networks that interact in a well-characterized manner (Fox and Raichle, 2007). A complementary measure of connectivity is the fractional Amplitude of Low Frequency Fluctuations (fALFF), which evaluates the amplitude of this spontaneous neural activity. As resting-state functional magnetic resonance imaging (RSfMRI) does not require an active task, it is especially practical in populations that may find traditional functional MRI tasks difficult to perform (Fox and Raichle, 2007).

Alterations within cognitive networks and in the relationships between those networks have been reported in patients with schizophrenia (for review, see Whitfield-Gabrieli and 
Ford (2012)). In medication-naïve and unmedicated patients, elevated dopamine turnover and release have been documented (Laruelle et al, 1996) and found to be predictive of good treatment response (Abi-Dargham et al, 2000). Long-term antipsychotic treatment appears to modulate FC patterns (Lui et al, 2010), but the early time course of those changes remains unknown. In healthy subjects, acute alterations in FC following L-dopa administration and haloperidol challenge have been reported (Cole et al, 2012b). Taken together, it is conceivable that abnormalities in FC patterns may not only set the stage for clinical response, but that modulation of connectivity patterns early in treatment could be an indicator for good response to treatment.

In this study, we use a seed-based approach to study the effects of risperidone on FC of the ventral tegmental area (VTA), the source of mesocorticolimbic dopaminergic projections thought to be critical for antipsychotic drug action (for review, see Goto and Grace (2007)). Given that mammalian studies report heavy dopaminergic innervation to the striatum, most regions of the frontal cortex and many temporal and parietal areas (Gaspar et al, 1989), it is plausible that antipsychotic drugs affect networks that are functionally connected to the VTA. Patients were scanned twice: (1) while unmedicated (baseline) and (2) after 1 week of treatment, in an attempt to elucidate the functional correlates of treatment-induced changes before clinical response is apparent. We hypothesize that unmedicated patients with schizophrenia exhibit significant alterations in VTA/midbrain connectivity and in fALFF compared with healthy controls, and that treatment elicits an early change in VTA/midbrain connectivity and fALFF. We also hypothesize that pre-treatment connectivity abnormalities are predictive of patient treatment response.

\section{MATERIALS AND METHODS}

\section{Participants}

Thirty-one unmedicated patients with schizophrenia were recruited at the University of Alabama at Birmingham (UAB). Diagnosis was based on clinical evaluation and medical records, and was confirmed by the Diagnostic Interview for Genetic Studies (DIGS; Nurnberger et al, 1994). All patients were off antipsychotic medications for at least 10 days (none had been treated with depot antipsychotics); medication was not discontinued to meet this criterion. Additionally, 23 healthy controls were recruited by newspaper and flyer advertisements. The UAB Institutional Review Board approved the study, and written informed consent was obtained after patients were deemed competent to provide consent (Carpenter et al, 2000).

Healthy controls had no current or lifetime Axis I disorders in themselves and first-degree relatives and had no history of psychotropic medication exposure. Exclusion criteria were having neurological disorders, use of medications known to affect brain function, serious medical conditions, pregnancy, substance abuse or dependence (except for nicotine) within 6 months of enrollment, history of head trauma with loss of consciousness, and MRI contraindications.

\section{Participant Dropout}

A total of 10 patients (5 did not tolerate scanning environment, 1 due to poor scan quality, 4 were lost to follow-up after initial scan) and 2 controls (both due to poor scan quality) were excluded, leaving 21 patients and 21 controls for analyses. Six patients missed clinical assessments at week 6; treatment response was defined using week 5 values for three patients, and response could not be determined for the others because they dropped out before week 5 .

\section{Experimental Design}

Healthy controls were scanned once; patients were scanned while unmedicated (baseline scan), and then entered into a 6-week trial with risperidone (flexible dosing regimen). During each weekly meeting, medication was counted to ensure compliance. A second scan was performed after 1 week of treatment. Symptom severity was assessed weekly using the Brief Psychiatric Rating Scale (BPRS; Overall and Gorham, 1962). Supplementary Figure S1 shows BPRS values for all participants across time. Cognitive functions were assessed at baseline using the Repeatable Battery for the Assessment of Neuropsychological Status (RBANS; Randolph et al, 1998). Treatment response was quantified as the percent change in BPRS scores from baseline to week 6 . To compare demographic and psychometric variables between groups, we performed independent $t$-tests and $\chi^{2}$-tests.

\section{MRI Acquisition}

All imaging was performed on a $3 \mathrm{~T}$ head-only scanner (Magnetom Allegra, Siemens Medical Solutions, Erlangen, Germany), equipped with a circularly polarized transmit/ receive head coil. RSfMRI scans were acquired during a 5-min gradient recalled echo-planar imaging sequence (repetition time/echo time $(\mathrm{TR} / \mathrm{TE})=2100 / 30 \mathrm{~ms}$, flip angle $=70^{\circ}$, field of view $=24 \times 24 \mathrm{~cm}^{2}, 64 \times 64$ matrix, 4 -mm slice thickness, 1-mm gap, 26 axial slices, 150 acquisitions). During the scan, participants were instructed to keep their eyes open and stare passively ahead. High-resolution structural scans were acquired using the 3-dimensional T1-weighted magnetization-prepared rapid acquisition gradient-echo sequence (TR/ $\mathrm{TE} /$ inversion time $(\mathrm{TI})=2300 / 3.93 / 1100 \mathrm{~ms}$, flip angle $=12^{\circ}$, $256 \times 256$ matrix, $1-\mathrm{mm}$ isotropic voxels). All MRI scans were reviewed for abnormalities by a qualified neuroradiologist.

\section{FC Analysis}

Using the statistical parametric mapping package SPM8 (Wellcome Trust Centre for Neuroimaging, London, UK), RSfMRI data were slice timing corrected, realigned using rigid-body motion transforms, co-registered to the highresolution structural scan, normalized to Montreal Neurologic Institute (MNI) space, resliced to $1.5 \mathrm{~mm}^{3}$ during normalization, and spatially smoothed with a $6-\mathrm{mm}$ at fullwidth half-maximum three-dimensional Gaussian kernel. Normalization was employed using the diffeomorphic anatomical registration using exponentiated lie algebra algorithm (DARTEL; Ashburner, 2007) to create a unique group template. To assess subjects' motion effects on FC analysis, mean absolute displacement of the brain from each 
time frame to the next was calculated. Displacements were not significantly different for patients at baseline (absolute displacement $0.24 \pm 0.28 \mathrm{~mm}$; mean $\pm \mathrm{SD}$ ) compared with week $1(0.17 \pm 0.14 \mathrm{~mm} ; t=-0.96 ; \mathrm{df}=40 ; p=0.26)$ or to controls $(0.16 \pm 0.07 \mathrm{~mm} ; t=-1.26 ; \mathrm{df}=40 ; p=0.22)$ or between patients at week 1 and controls $(t=0.43$; df $=40$; $p=0.66)$.

To remove potential sources of noise from the data, a nuisance regression was first conducted using the six motion parameters identified during the realignment step and using their first derivatives as regressors. Then, a stepwise data 'scrubbing' procedure was performed on the data in order to reduce the effects of time points severely contaminated by motion (Power et al, 2012a). Framewise displacements from each time point, $i$, to the next $\left(\mathrm{FD}_{i}\right)$ were computed from the six realignment parameters from SPM8. A radius $r=50 \mathrm{~mm}$ (approximately the mean distance from the center of MNI space to the cortex), was used to convert angle rotations (radians) to displacements $(\mathrm{mm})$. Time points with $\mathrm{FD}_{i}>0.5 \mathrm{~mm}$ were considered to be severely contaminated with motion or other artifact. These contaminated time points were first interpolated before bandpass filtering of the data $(0.009<\mathrm{f}<0.08 \mathrm{~Hz}$ ) (Carp, 2011 ), and then excluded from subsequent analyses (Power et al, 2012b). Following data 'scrubbing', a principle component analysis was used to extract the components of white matter and cerebral spinal fluid necessary to explain $90 \%$ of signal variance from those regions. These extracted components were used as regressors in a second nuisance regression (Behzadi et al, 2007).

Connectivity analyses. A spherical region of interest with radius $=3 \mathrm{~mm}$ containing 46 voxels (cubic volume $=0.155$ $\mathrm{ml}$ ) was selected to represent the VTA/midbrain, centered at MNI coordinates $0,-16,-7$ ( $\mathrm{Gu}$ et al, 2010). We centered a 6-mm spherical region of interest with 294 voxels (cubic volume, $1.02 \mathrm{ml}$ ) in the gray matter of the left middle occipital cortex (MOC) for use as a control region (MNI coordinates: $-18,-95,0)$. The first eigenvariate (extracted using singular value decomposition of the time series across all the voxels within each ROI, implemented in the REX toolbox) was used to describe the maximal variance of the RSfMRI time series explained from each region and correlated to the time series of all other voxels in the brain to produce a FC map (units of Pearson's $r$ correlations). These maps were converted to normally distributed values using Fisher's $r$-to- $Z$ transform before group comparisons.

fALFF analyses. fALFF was calculated using the REST toolbox, version 1.8, as previously described (Song et al, 2011). After preprocessing and the removal of linear trends, the RSfMRI time series for each voxel was transformed into the frequency domain using the fast-Fourier transform. The square root of the power spectra at each frequency was used to derive amplitudes, and then the sum of the amplitudes across $0.01-0.08 \mathrm{~Hz}$ was divided by that of the entire frequency range $(0-0.25 \mathrm{~Hz})$. This resulted in a fALFF map for each participant at each time point for group comparisons.
Statistical analyses. Group-level FC maps were obtained by performing one-sample $t$-tests on each group's participant-level FC maps. Group differences in FC were assessed using a two-sample $t$-test on the groups' participant-level FC maps. An analysis of covariance (ANCOVA) was performed to examine the relationship of pre-treatment and 1-week post-treatment FC with treatment response. An ANCOVA was performed to examine the relationship of change in VTA/midbrain to thalamus FC with treatment response. All analyses were corrected for multiple comparisons at the cluster level using the false discovery rate (FDR; Chumbley and Friston, 2009) and are reported at $p_{\mathrm{FDR}}<0.05$.

\section{Reliability mapping of VTA/Midbrain connectivity} correlation to treatment response. To visually inspect reliability of significant regions in connectivity analyses, we used a 'leave-one-out' repeated analysis, as previously described (Skidmore et al, 2013). Briefly, statistical analyses are repeated $N$ times (18 in our study, for the 18 patients included in this analysis), each time leaving out one subject. For each of the 18 analyses, data are thresholded so that voxels are assigned a value of 1 when $p_{\text {FDR }}<0.05$ or a value of 0 when $p_{\text {FDR }} \geqslant 0.05$. Finally, the 18 thresholded analyses were summed to create a 'reliability map' showing the voxel-by-voxel reliability of statistical significance.

\section{RESULTS \\ Demographics}

No significant differences between patients and controls in age, sex, parental socioeconomic status, smoking status, or cigarette consumption were observed (Table 1).

\section{Behavioral Results}

Controls scored significantly higher on the RBANS. Six patients were antipsychotic drug naïve, and 15 had previously been treated with antipsychotics (Table 1). No difference in symptom severity at baseline $(t=-1.45$; $\mathrm{df}=19 ; p=0.16)$ or in treatment response between antipsychotic naïve and previously medicated patients was observed $(t=0.97 ; \mathrm{df}=16 ; p=0.64)$.

\section{VTA/Midbrain Connectivity}

Connectivity patterns in controls. In controls, analysis revealed significant VTA/midbrain connectivity to neighboring regions of the midbrain, striatum, thalamus, bilateral hippocampi, amygdala, anterior and middle cingulate cortex, middle and inferior frontal gyri, precuneus, calcarine sulcus, superior parietal gyrus, and insula (Supplementary Figure S2, Supplementary Table S1; Supplementary Tables S2 and S3 show connectivity in pre- and post-treatment schizophrenia). This analysis also revealed significant MOC connectivity to neighboring regions of the cuneus, precuneus, cerebellum, middle cingulate cortex, and insula (Supplementary Figure S3). 
Table I Demographic Characteristics and Clinical Measures for Participants $^{a}$

\begin{tabular}{lccrc}
\hline & $\begin{array}{c}\text { Patients } \\
(\mathbf{n}=\mathbf{2} \mathbf{I})\end{array}$ & $\begin{array}{c}\text { Controls } \\
(\mathbf{n}=\mathbf{2} \mathbf{I})\end{array}$ & $\boldsymbol{t} / \boldsymbol{\chi}^{\mathbf{2}}$ & $\boldsymbol{p}$-value \\
\hline Demographic characteristics & & & & \\
Age (years) & $36.0 \pm 10.2$ & $35.5 \pm 10.4$ & -0.15 & 0.88 \\
Sex (male/female) & $17 / 4$ & $11 / 10$ & 1.62 & 0.29 \\
Parental SES & $7.4 \pm 4.9$ & $9.9 \pm 4.0$ & 0.02 & 0.98 \\
Smoking status (Y/N) & $19 / 2$ & $18 / 3$ & 1.42 & 0.44 \\
Smoking (packs per day) & $0.73 \pm 0.5$ & $0.96 \pm 0.6$ & 1.36 & 0.18
\end{tabular}

\section{Diagnosis}

Schizophrenia

Schizoaffective disorder

Illness characteristics

Illness duration (years)

First episode $(\mathrm{Y} / \mathrm{N})$

$\begin{array}{cc}13.3 \pm 9.5 & - \\ 6 / 15 & -\end{array}$

Prior antipsychotic treatment

Antipsychotic naïve ( $\mathrm{Y} / \mathrm{N})$

Antipsychotic-free

interval (mo.)

BPRS at baseline

Total score

Positive symptom subscale

$44.4 \pm 0.6$

Negative symptom subscale

$11.9 \pm 4.0$

$6.5 \pm 2.3$

BPRS at week 6

Total score

Positive symptom subscale

Negative symptom subscale

$$
\begin{array}{r}
27.4 \pm 7.8 \\
5.4 \pm 2.3 \\
5.1 \pm 2.4
\end{array}
$$

RBANS

$\begin{array}{lllll}\text { Total index } & 73.8 \pm 14.3 & 98.9 \pm 13.7 & 5.82 & <0.01 \\ \text { Immediate memory } & 79.8 \pm 20.0 & 98.1 \pm 9.8 & 3.77 & <0.01 \\ \text { Visuospatial } & 69.9 \pm 14.7 & 92.1 \pm 17.4 & 4.46 & <0.01 \\ \text { Language } & 87.0 \pm 9.9 & 101.4 \pm 14.3 & 3.79 & <0.01 \\ \text { Attention } & 82.4 \pm 18.9 & 107.5 \pm 18.5 & 4.35 & <0.01 \\ \text { Delayed memory } & 77.3 \pm 17.3 & 96.8 \pm 7.5 & 4.86 & <0.01\end{array}$

Abbreviations: BPRS, Brief Psychiatric Rating Scale; N, no; RBANS, Repeated Battery for the Assessment of Neuropsychological Status; SES, socioeconomic status; $Y$, yes.

${ }^{a}$ Mean \pm SD are shown unless indicated otherwise.

${ }^{b}$ Ranks determined from the Diagnostic Interview for Genetic Studies, reported on I-18 scale; higher rank (lower numerical value) corresponds to higher socioeconomic status. Information was unavailable for four patients.

Reduced pre-treatment VTA/midbrain connectivity in schizophrenia. Compared with controls, regions including precuneus, inferior parietal cortex, dorsal anterior cingulate cortex (dACC), middle cingulate cortex, superior, middle, and inferior frontal gyri, insula, thalamus, hippocampus, caudate, putamen and cerebellum demonstrated weaker pre-treatment VTA/midbrain connectivity (Figure 1a, Table 2) in schizophrenia. No regions exhibited higher pre-treatment VTA/ midbrain connectivity in schizophrenia compared with controls. Finally, no differences in pre-treatment and control MOC connectivity were observed.

Increased VTA/midbrain connectivity after 1 week of treatment in schizophrenia. Compared with pre-treatment values, a significant increase in 1-week post-treatment $\mathrm{VTA} /$ midbrain connectivity to bilateral regions of the thalamus was observed (Figure 1b, Table 2). However, pre-treatment VTA/midbrain connectivity strength to the thalamus was not correlated with treatment response (Supplementary Figure S4). The change in connectivity strength between the VTA/midbrain and thalamus before and after treatment was not correlated with treatment response $(r=0.096, p=0.703)$. No regions exhibited decreased VTA/midbrain connectivity post treatment. No differences in pre-treatment and post-treatment MOC connectivity were observed.

Treatment response correlated with pre-treatment VTA/ midbrain connectivity in schizophrenia. Treatment response was positively correlated to pre-treatment VTA/ midbrain connectivity strength in the dACC and supplementary motor cortex, and negatively correlated to pretreatment VTA/midbrain connectivity strength in regions comprising the default mode network (DMN), including the ventromedial prefrontal cortex, posterior cingulate cortex, precuneus, and lateral parietal cortex (Figure 2, Supplementary Table S4; for relationship using residualized BPRS scores, see Supplementary Figure S5). No relationship was observed between treatment response and 1-week posttreatment VTA/midbrain connectivity, pre- or 1-week posttreatment MOC connectivity.

Reliability analyses. From the reliability maps (Supplementary Figure S6), we can observe that some specific cortical regions have varying reliability: for positive correlations, two regions are reliable (dACC and the supplementary motor area), and for negative correlations, three regions are reliable (medial prefrontal cortex, precuneus/posterior cingulate cortex, and left lateral parietal cortex). Interestingly, these regions are the key nodes of the DMN. Additionally, our results indicate that negative correlations are generally more reliable than positive correlations.

\section{fALFF Results}

Differences in unmedicated patients and controls. Compared with controls, pre-treatment patients showed decreased fALFF in regions including the precuneus, posterior cingulate cortex, anterior and middle cingulate cortices, and medial prefrontal cortex. Pre-treatment patients showed increased fALFF only in the cerebellum compared with controls (Figure 1c, Table 3).

Increased fALFF following 1 week of treatment. After 1 week of treatment, patients showed increased fALFF in the dorsolateral prefrontal cortex extending to the fronto-insular 
a VTA/midbrain functional connectivity: Controls > Pre-treatment patients
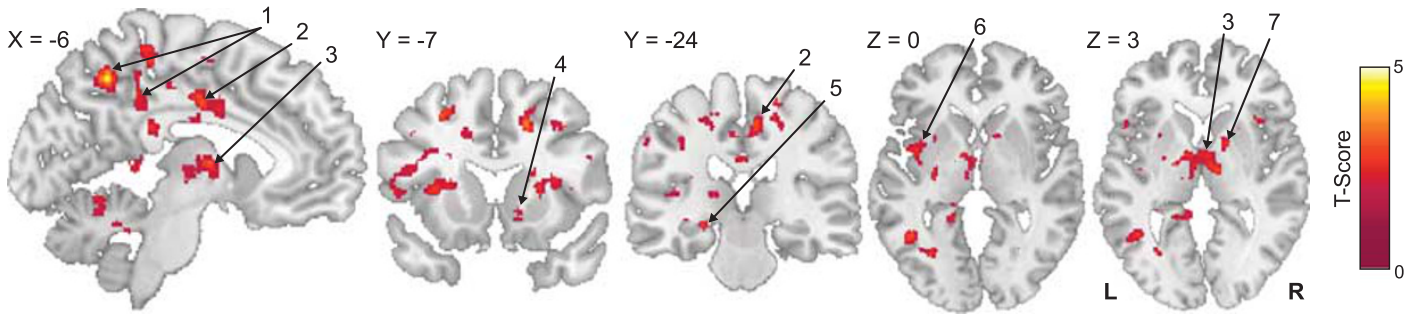

b VTA/midbrain functional connectivity: Pre-treatment < Post-treatment patients
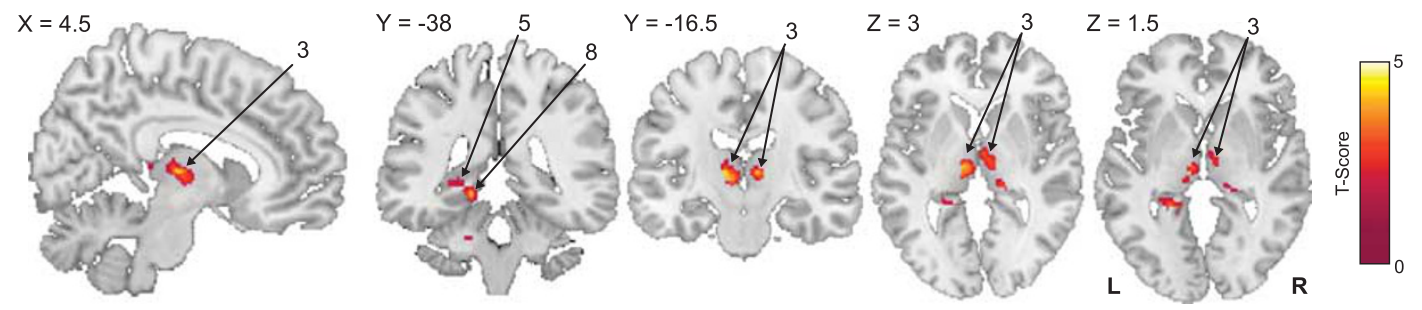

C

fALFF: Controls - Pre-treatment Patients
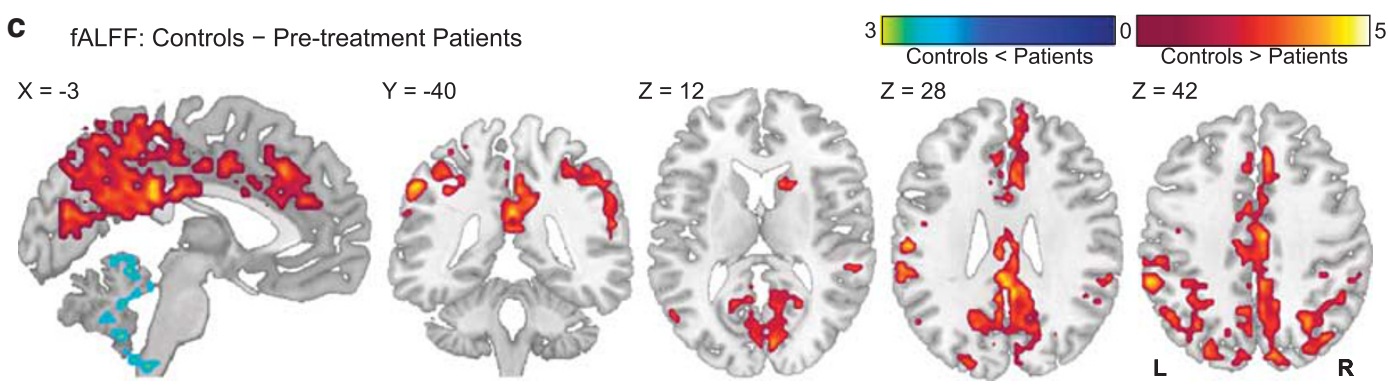
$Z=42$

d fALFF: Pre-treatment - Post-treatment Patients
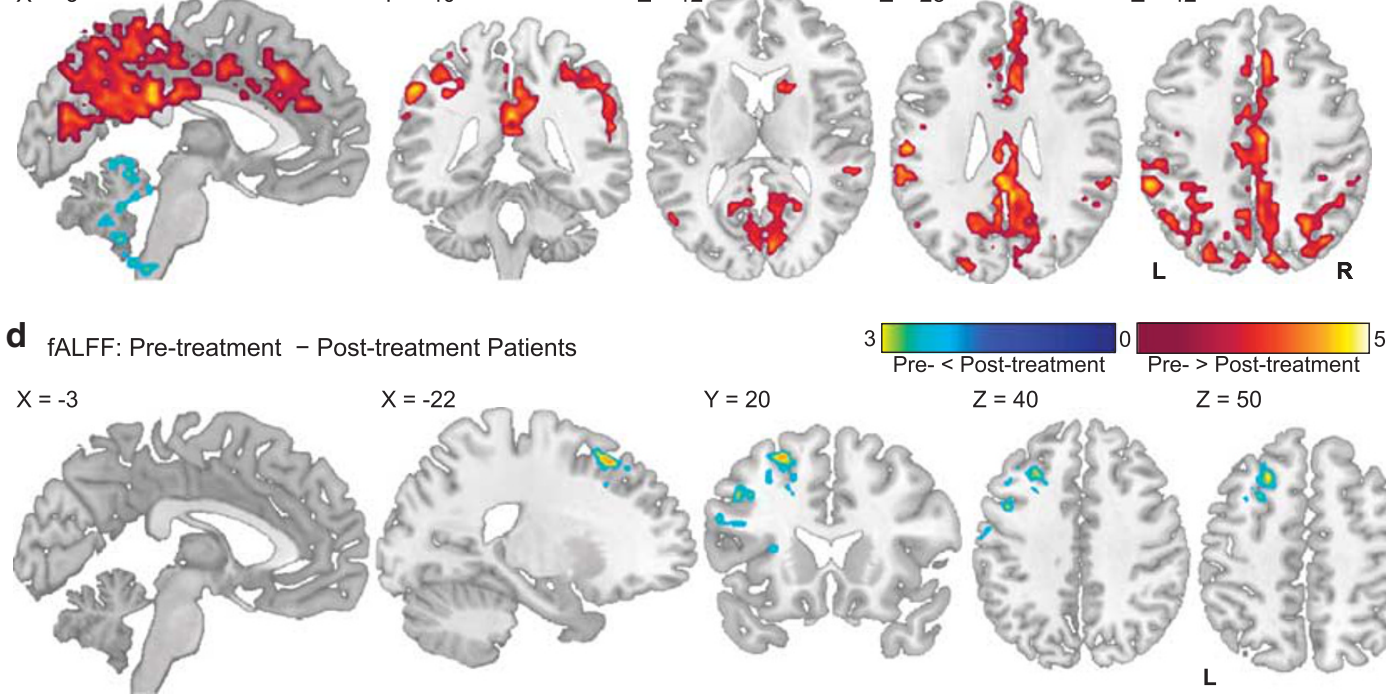

$Z=40 \quad Z=50$

Figure I Group differences in resting-state functional connectivity. (a) Regions showing lower ventral tegmental area (VTA)/midbrain connectivity in controls $(n=21)$ compared with pre-treatment unmedicated patients with schizophrenia $(n=21$; two-tailed two-sample $t$-test corrected with the false discovery rate (FDR) at PFDR $<0.05$ ). (b) Regions showing increased VTA/midbrain connectivity after I week of antipsychotic treatment $(n=21$; two-tailed two-sample $t$-test corrected with the FDR at PFDR <0.05). (c) Regions where fractional Amplitude of Low Frequency Fluctuations (fALFF) differs significantly in pre-treatment unmedicated patients with schizophrenia $(n=21)$ compared with controls $(n=21$; two-tailed two-sample $t$-test corrected with the FDR at PFDR <0.05). (d) Regions showing increased fALFF after I week of antipsychotic treatment ( $n=21$; two-tailed two-sample $t$-test corrected with the FDR at PFDR <0.05). For all panels, data are overlaid on a single-subject high-resolution TI image of an extracted brain in neurologic convention (left on left). Labeled regions: I, precuneus/posterior cingulate cortex; 2, middle cingulate cortex extending to the dorsal anterior cingulate cortex; 3, thalamus; 4, nucleus accumbens; 5, hippocampus; 6, insula; 7, pallidum; 8, parahippocampus.

regions (Figure 1d, Table 3). None of the regions exhibited decreased AALFF with treatment.

\section{DISCUSSION}

To our knowledge, this is the first time that VTA/midbrain FC in patients with schizophrenia has been studied during the resting state. In this study, we contrasted VTA/midbrain
FC in unmedicated patients to that of healthy controls, and investigated changes of connectivity patterns within 1 week of antipsychotic treatment. As hypothesized, we found an abnormal reduction in VTA/midbrain connectivity to many cortical and subcortical areas in unmedicated patients with schizophrenia compared with controls; deficits in connectivity to the thalamus were restored after 1 week of treatment. Interestingly, VTA/midbrain connectivity to the DMN and dACC at baseline were correlated with clinical 
Table 2 Regions Showing Differences in VTA/midbrain Functional Connectivity between Groups

\begin{tabular}{|c|c|c|c|c|c|c|c|c|}
\hline \multirow[t]{2}{*}{ Brain regions } & \multirow[t]{2}{*}{ Voxels in cluster } & \multirow[t]{2}{*}{ Hem. } & \multirow[t]{2}{*}{ Voxels in region } & \multicolumn{3}{|c|}{ Peak coordinates $^{\mathrm{a}}$} & \multirow[t]{2}{*}{ Peak $t$} & \multirow[t]{2}{*}{$P_{F D R}{ }^{b}$} \\
\hline & & & & $x$ & $Y$ & $z$ & & \\
\hline \multicolumn{9}{|l|}{$H C>S Z O$} \\
\hline L Precuneus/parietal/occipital & 2583 & & & -30 & -69 & 14 & 6.19 & 0.001 \\
\hline Superior parietal gyrus & & L & 376 & & & & & \\
\hline Inferior parietal gyrus & & L & 545 & & & & & \\
\hline Precuneus & & L & 333 & & & & & \\
\hline Middle occipital gyrus & & L & 304 & & & & & \\
\hline Middle cingulate/frontallinsula & 8185 & & & 6 & -27 & 50 & 5.34 & $<0.001$ \\
\hline Middle frontal gyrus & & L & 411 & & & & & \\
\hline Inferior frontal gyrus & & L & 505 & & & & & \\
\hline Precentral gyrus & & B & 387 & & & & & \\
\hline Superior temporal gyrus & & L & 138 & & & & & \\
\hline Middle temporal gyrus & & L & 205 & & & & & \\
\hline Putamen/pallidum & & L & 403 & & & & & \\
\hline Insula & & L & 490 & & & & & \\
\hline Middle cingulate cortex & & L & 1230 & & & & & \\
\hline$R$ Frontal/anterior cingulate & 1345 & & & -21 & -6 & 4 & 4.05 & 0.014 \\
\hline Superior frontal gyrus & & R & 416 & & & & & \\
\hline Middle frontal gyrus & & $\mathrm{R}$ & 329 & & & & & \\
\hline Anterior cingulate cortex & & $\mathrm{R}$ & 166 & & & & & \\
\hline B Thalamus & 1086 & & & 22 & -54 & -24 & 3.91 & 0.033 \\
\hline Thalamus & & B & 562 & & & & & \\
\hline R Fusifrom/cerebellum & 1374 & & & -22 & -45 & -16 & 3.82 & 0.014 \\
\hline Lingual/fusiform gyrus & & R & 635 & & & & & \\
\hline Cerebellum & & R & 659 & & & & & \\
\hline L Fusiform/cerebellum & 1704 & & & 15 & 62 & 9 & 3.81 & 0.007 \\
\hline Lingual/fusiform gyrus & & L & 424 & & & & & \\
\hline Parahippocampal gyrus & & L & 147 & & & & & \\
\hline Posterior cingulate cortex & & L & 69 & & & & & \\
\hline Cerebellum & & L & 584 & & & & & \\
\hline Vermis & & & 157 & & & & & \\
\hline R Frontal/caudate/putamen & 1544 & & & 15 & 62 & 9 & 3.69 & 0.010 \\
\hline Superior frontal gyrus & & $\mathrm{R}$ & 451 & & & & & \\
\hline Middle frontal gyrus & & R & 196 & & & & & \\
\hline Caudate nucleus & & $\mathrm{R}$ & 43 & & & & & \\
\hline Putamen & & $\mathrm{R}$ & 93 & & & & & \\
\hline Insula & & R & 174 & & & & & \\
\hline \multicolumn{9}{|l|}{$s Z 0<S Z 1$} \\
\hline Lingual/hippocampus/thalamus & 2062 & & & -10 & -15 & 4 & 4.51 & $<0.001$ \\
\hline Lingual/fusiform gyrus & & L & 299 & & & & & \\
\hline Hippocampus/parahippocampus & & L & 153 & & & & & \\
\hline Thalamus & & B & 670 & & & & & \\
\hline Cerebellum & & L & 488 & & & & & \\
\hline
\end{tabular}

Abbreviations: HC, healthy controls; Hem., hemisphere; L, left; R, right; B, bilateral; SZO, unmedicated patients with schizophrenia; SZI, patients after I week of antipsychotic drugs; VTA, ventral tegmental area.

${ }^{a}$ Reported in Montreal Neurologic Institute coordinates $(X, Y$, and $Z)$.

${ }^{\mathrm{b}}$ All comparisons were FDR-corrected at the cluster level for $p_{\mathrm{FDR}} \leqslant 0.05$. 
a

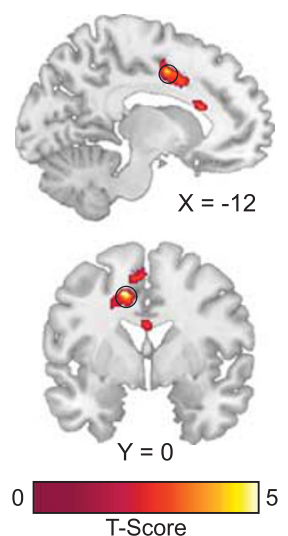

b

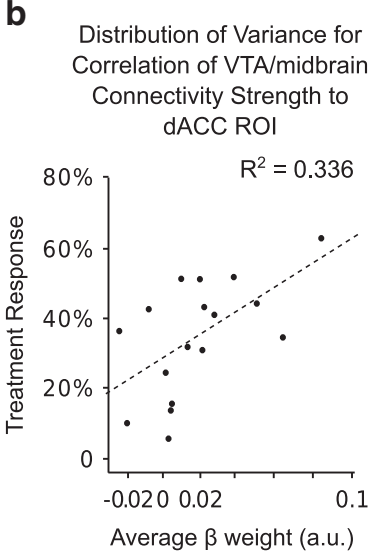

C

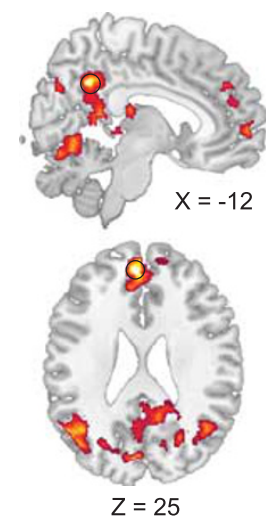

d

Distribution of Variance for Correlation of VTA/midbrain Connectivity Strength to $\mathrm{PCC} /$ Precuneus ROI

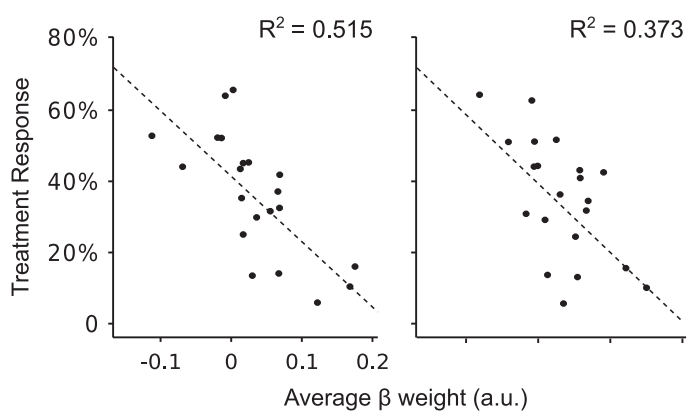

Figure 2 Relationship between pre-treatment ventral tegmental area (VTA)/midbrain functional connectivity and treatment response at 6 weeks. (a) Regions where pre-treatment VTA/midbrain functional connectivity shows a positive correlation with treatment response (analysis of covariance corrected with the false discovery rate (FDR) at PFDR <0.05). (b) Scatter plot showing pre-treatment VTA/midbrain functional connectivity to voxels in the dACC (3 mm sphere centered at $(-13,0,42)$, circled in black in panel a) vs treatment response (one outlier, identified as having VTA/midbrain to dACC connectivity strength more than 3 SD from the mean, was removed from analysis; $n=17$ ). (c) Regions where pre-treatment VTA/midbrain functional connectivity shows a negative correlation with treatment response (analysis of covariance corrected with the FDR at $P_{\mathrm{FDR}}<0.05$ ). (d) Scatter plots showing pre-treatment VTA/midbrain functional connectivity in the posterior cingulate cortex (PCC)/precuneus (3 mm sphere centered at (5, - 50, 24), circled in black in the sagittal slice (top) of panel c) and in the medial prefrontal cortex (MPFC; 3 mm sphere centered at ( - 6, 50, 24), circled in black in the axial slice (bottom) of panel c) plotted against treatment response. For panels a and c, results are overlaid on a single-subject high-resolution $\mathrm{TI}$ image of an extracted brain in neurologic convention (left on left).

response after 6 weeks. Changes in connectivity to the DMN and dACC during the first week of treatment were not significantly correlated with clinical response.

In controls, we found significant connectivity from VTA/ midbrain to the midbrain, striatum, thalamus, bilateral hippocampi, amygdala, anterior and middle cingulate cortex, middle and inferior frontal gyri, precuneus, calcarine sulcus, superior parietal gyrus, and insula. This is consistent with what has been reported in the literature in healthy participants of similar age range ( $\mathrm{Gu}$ et al, 2010; Tomasi and Volkow, 2012). Our findings are also consistent with previous studies on the anatomy of the mesocorticolimbic dopamine system in mammals. The VTA anatomically projects to the striatum, several cortical areas, thalamus, and amygdala; the very areas we find to be functionally connected to the VTA/midbrain (Perez-Costas et al, 2010). Importantly, VTA FC to these regions was confirmed to be highly reproducible and sensitive to disruption by disorders such as attention deficit hyperactivity disorder, highlighting its potential as biomarker of dopamine pathway dysfunction (Tomasi and Volkow, 2012).

Very few studies have evaluated FC patterns in unmedicated patients with schizophrenia. A combined structural/ RSfMRI study evaluated gray matter abnormalities in a relatively large group of antipsychotic naïve, first-episode patients. Three areas were identified to have gray matter deficits and subsequently defined as seed regions for FC analysis: the anterior cingulate cortex, superior temporal gyrus, and middle temporal gyrus. Although no abnormalities in connectivity patterns from these seeds were detected in comparison with healthy controls, temporo-putamen and temporo-precuneus connectivity levels were associated with symptom severity (Lui et al, 2009). In a subsequent study, the same group reported decreased ALFF in orbital medial frontal cortex and increased ALFF in the bilateral putamen in unmedicated, antipsychotic-naïve patients with schizophrenia relative to controls. Following 6 weeks of treatment, ALFF increased in the right middle frontal gyrus, right inferior parietal lobe, bilateral medial frontal cortex, right inferior frontal gyrus, left superior frontal gyrus, left superior temporal gyrus, and right caudate. In our study, we identified more extensive ALFF abnormalities in pretreatment, unmedicated patients compared with controls, notably in areas of the DMN such as the anterior cingulate cortex, posterior cingulate cortex, precuneus, and angular gyrus. Discrepancies between those findings may arise from differences in illness duration or previous antipsychotic exposure in patient populations studied, as the patients studied by Lui et al (2010) were antipsychotic naïve. One could speculate ALFF abnormalities may become more widespread with prolonged illness duration. Interestingly, ALFF increases after 1 week in our group overlap with those reported by Lui et al (2010) after 6 weeks of treatment; these areas include the superior, middle, and inferior frontal gyri.

Here, we demonstrate VTA/midbrain connectivity to the thalamus is similar to that of healthy controls after 1 week of antipsychotic treatment. Thalamic abnormalities have been reported in multiple imaging and postmortem studies (for review, see Clinton and Meador-Woodruff, 2004), suggesting alterations in thalamic volumetric measures (Shepherd et al, 2012), deformities of thalamic shape (Csernansky et al, 2004), reduction of $\mathrm{N}$-acetyl aspartate, a putative marker of neuronal integrity (Kraguljac et al, 2012), cellular abnormalities, particularly in the dorsomedial nucleus (Meador-Woodruff et al, 2003), and reduced glutamatergic gene expression in thalamocortical neurons (Sodhi et al, 2011). Congruent with our results, antipsychotic drugs have been shown to affect thalamic metabolism (Holcomb et al, 1996) and functions (Lahti et al, 2005). 
Table 3 Regions Showing Differences in fALFF in Participant Groups

\begin{tabular}{|c|c|c|c|c|c|c|c|c|}
\hline \multirow[t]{2}{*}{ Brain regions } & \multirow[t]{2}{*}{ Voxels in cluster } & \multirow[t]{2}{*}{ Hem. } & \multirow[t]{2}{*}{ Voxels in region } & \multicolumn{3}{|c|}{ Peak coordinates $^{\mathrm{a}}$} & \multirow[t]{2}{*}{ Peak $t$} & \multirow[t]{2}{*}{$P_{F D R}{ }^{b}$} \\
\hline & & & & $x$ & $Y$ & $z$ & & \\
\hline \multicolumn{9}{|l|}{$H C>S Z O$} \\
\hline DMN/frontal & 3587 & & & 3 & -48 & 48 & 4.61 & $<0.001$ \\
\hline Superior frontal gyrus & & B & 111 & & & & & \\
\hline Precentral gyrus & & L & 35 & & & & & \\
\hline Postcentral gyrus & & B & 196 & & & & & \\
\hline Superior temporal gyrus & & $\mathrm{R}$ & 32 & & & & & \\
\hline Superior occipital gyrus & & L & 77 & & & & & \\
\hline Superior parietal gyrus & & B & 274 & & & & & \\
\hline Inferior parietal gyrus & & B & 344 & & & & & \\
\hline Angular gyrus & & B & 128 & & & & & \\
\hline Supramarginal gyrus & & B & 111 & & & & & \\
\hline Precuneus & & B & 747 & & & & & \\
\hline Superior occipital gyrus & & $\mathrm{R}$ & 54 & & & & & \\
\hline Middle occipital gyrus & & B & 69 & & & & & \\
\hline Cuneus & & B & 164 & & & & & \\
\hline Calcarine fissure & & L & 230 & & & & & \\
\hline Lingual gyrus & & $\mathrm{R}$ & 79 & & & & & \\
\hline Anterior cingulate cortex & & B & 118 & & & & & \\
\hline Middle cingulate cortex & & B & 406 & & & & & \\
\hline Posterior cingulate cortex & & L & 117 & & & & & \\
\hline \multicolumn{9}{|l|}{$H C<S Z O$} \\
\hline Cerebellum & 359 & & & -18 & -51 & -42 & 3.26 & 0.029 \\
\hline Cerebellum & & B & 136 & & & & & \\
\hline Vermis & & & 26 & & & & & \\
\hline \multicolumn{9}{|l|}{$s Z 0<S Z I$} \\
\hline L Frontal/precentral & 317 & & & -21 & 24 & 51 & 5.72 & \\
\hline Inferior frontal gyrus & & L & 71 & & & & & \\
\hline Middle frontal gyrus & & L & 127 & & & & & \\
\hline Precentral gyrus & & L & 38 & & & & & \\
\hline
\end{tabular}

Abbreviations: fALFF, Fractional Amplitude of Low Frequency Fluctuations; Hem., hemisphere; L, left; R, right; B, bilateral; SZO, unmedicated patients with schizophrenia; SZI, patients after I week of antipsychotic drugs.

a Reported in Montreal Neurologic Institute coordinates ( $X, Y$, and $Z$ ).

${ }^{\mathrm{b}}$ All comparisons were FDR-corrected at the cluster level for $p_{\mathrm{FDR}} \leqslant 0.05$.

Specifically, Liddle et al (2000) identified changes in glucose metabolism in the thalamus of antipsychotic drug-naïve patients after a single dose of risperidone. Surprisingly, in agreement with our findings, this alteration was not predictive of subsequent clinical response (Liddle et al, 2000). Although risperidone seems to modulate both thalamic metabolism and FC to the thalamus, it is intriguing that these early changes do not appear to be related to clinical response. It is possible that restoration of VTA/ midbrain to thalamus connectivity alone may not improve positive symptoms but rather set the stage for restoration of connectivity deficits between the VTA/midbrain and cortical areas that are more directly related to clinical improvement; this needs to be further investigated by longitudinal imaging studies. The importance of VTA/ midbrain connectivity to cortical areas for clinical response is further highlighted by our findings that baseline VTA/ midbrain connectivity to the AACC and to areas of the DMN is related to eventual clinical improvement. It is notable that patients with the least disrupted FC between VTA/midbrain and specifically the dACC appear more likely to respond to antipsychotics. Animal studies have shown that dopaminergic neurons projecting from the VTA to the prefrontal cortex and nucleus accumbens are differentially regulated (Del Arco and Mora, 2009) and postmortem studies have indicated a reduced density of mesocortical projections to the prefrontal cortex in schizophrenia (Akil et al, 1999). Collectively, data suggest structural and functional integrity 
between the VTA/midbrain and AACC are pivotal for a favorable treatment response. Both the VTA/midbrain and dACC are components of the salience network, which is involved in distinguishing the relative importance of information (Seeley et al, 2007). Salience encoding is thought to occur in part via dopaminergic projections from the VTA (for review, see Salamone and Correa (2012)). Misattributed salience mediated by increased dopamine has been proposed as a potential mechanism for the positive symptoms of schizophrenia (Kapur, 2003). It is possible that a medication-induced decrease in dopaminergic activity within this network alleviates symptoms by restoring salience attribution.

Our results also demonstrate a negative correlation between treatment response and VTA/midbrain connectivity to regions of the DMN (rostral anterior cingulate cortex, ventromedial prefrontal cortex, precuneus, posterior cingulate cortex, and bilateral lateral parietal cortex) (Raichle et al, 2001). The DMN is the most prominent network active during rest, as has been characterized in multiple studies (for review, see Buckner et al (2008); Whitfield-Gabrieli and Ford, 2012). Its activity has been associated with introspective tasks, including self-reflection and social cognition (Buckner et al, 2008). Neuroimaging studies combining RSfMRI and positron-emission tomography suggest dopamine modulates the DMN (Cole et al, 2012a). Additionally, DMN abnormalities have consistently been reported in schizophrenia (Whitfield-Gabrieli and Ford, 2012). Restingstate studies have demonstrated that the activity of taskpositive networks, such as the salience network, is negatively coupled with the activity of the DMN (Fox et al, 2005); thus, activation in one is proportional to deactivation in the other. It has been proposed that in schizophrenia, altered activity of the DMN reduces the separation between networks, which could contribute to an inability to distinguish between internal and external stimuli (Whitfield-Gabrieli and Ford, 2012).

Sambataro et al (2010) evaluated the effects of treatment with olanzapine on FC of the DMN and reported increases in connectivity between this network and the ventromedial prefrontal cortex between weeks 4 and 8 of treatment, but unlike our study, their scans were not obtained in unmedicated patients. These data suggest changes in FC are observed beyond those seen in the early stages of treatment.

Important limitations need to be considered when interpreting these data. The sequence used for RSfMRI has a relatively low spatial resolution $\left(\sim 4 \mathrm{~mm}^{3}\right)$. Because small brainstem structures are difficult to localize, we cannot be certain that our seed region was exclusively confined to the VTA in all participants; we therefore refer to this region as 'VTA/midbrain'. RSfMRI is prone to contamination by non-neural signals, such as movement or heart rate. As part of the current analysis, well-validated preprocessing techniques and motion 'scrubbing' were used to reduce spurious correlations due to non-neural signals (Power et al, 2012a). However, there was a non-significant reduction in average head motion before and after treatment, and so spurious correlations cannot be definitively ruled out. FC is a bidirectional measure and cannot determine causal relationships between brain regions. FC can also occur between two regions not directly structurally connected and should not be interpreted to reflect direct anatomical projections (Damoiseaux and Greicius, 2009). Therefore, additional studies of structural and/or effective connectivity are necessary to more thoroughly characterize the relationship between connectivity and treatment response. Interpretation of results may be more difficult because comparisons between patient groups and correlation with treatment response were assessed on the whole brain (rather than constrained to regions found to be connected to the VTA/midbrain), as the relevance of these changes is unclear. Notably, the absence of a placebo group in our study limits interpretation of longitudinal findings; we are not able to definitively attribute changes in VTA/ midbrain connectivity to antipsychotic effects (rather than to the natural course of the disorder).

In summary, we describe significant differences in seedbased FC of the VTA/midbrain and in fALFF, a complementary measure of spontaneous neural activity. Deficits in VTA/midbrain connectivity to the thalamus were restored after 1 week of treatment with risperidone, suggesting that these deficits may be associated with known abnormalities in dopaminergic neurotransmission and modulated by antipsychotic treatment. Further studies are needed to clarify the utility of this potential biomarker in predicting clinical response to antipsychotic medication.

\section{FUNDING AND DISCLOSURE}

This research was supported by the National Institutes of Health (NIH; R01MH081014) and the University of Alabama Health Services Foundation General Endowment Fund Scholar Award. Parts of this work were supported by the research computing resources acquired and managed by the UAB Information Technology Research Computing division (from the National Science Foundation and NIH). Medication for this study was donated by Janssen Pharmaceuticals, Inc. Previously, Dr Lahti has received an investigatorinitiated research grant from Pfizer. Hadley was supported by the UAB Medical Scientist Training Program (NIH 5T32GM008361). Dr Visscher was supported by funding from the Dana Foundation, the UAB Center for Clinical and Translational Science (NIH UL1TR000165), the Vision Science Research Center (NIH P30EY003039), Civitan International Research Center, McKnight Brain Research Foundation, Edward R. Roybal Center for Translational Research on Aging and Mobility (NIH 2P30AG022838), and the UAB Comprehensive Center for Healthy Aging. Dr Kraguljac has received research funding from the UAB Department of Psychiatry and Behavioral Neurobiology. Dr Nenert, Dr Bolding, Dr Skidmore, and Mr White declare no potential conflict of interest.

\section{REFERENCES}

Abi-Dargham A, Rodenhiser J, Printz D, Zea-Ponce Y, Gil R, Kegeles LS et al (2000). Increased baseline occupancy of D2 receptors by dopamine in schizophrenia. Proc Natl Acad Sci USA 97: 8104-8109.

Agid O, Kapur S, Arenovich T, Zipursky RB (2003). Delayed-onset hypothesis of antipsychotic action: a hypothesis tested and rejected. Arch Gen Psychiatry 60: 1228-1235. 
Akil M, Pierri JN, Whitehead RE, Edgar CL, Mohila C, Sampson AR et al (1999). Lamina-specific alterations in the dopamine innervation of the prefrontal cortex in schizophrenic subjects. Am J Psychiatry 156: 1580-1589.

Ashburner J (2007). A fast diffeomorphic image registration algorithm. Neuroimage 38: 95-113.

Behzadi Y, Restom K, Liau J, Liu TT (2007). A component based noise correction method (CompCor) for BOLD and perfusion based fMRI. Neuroimage 37: 90-101.

Biswal B, Yetkin FZ, Haughton VM, Hyde JS (1995). Functional connectivity in the motor cortex of resting human brain using echo-planar MRI. Magn Reson Med 34: 537-541.

Buckner RL, Andrews-Hanna JR, Schacter DL (2008). The brain's default network: anatomy, function, and relevance to disease. Ann N Y Acad Sci 1124: 1-38.

Carp J (2011). Optimizing the order of operations for movement scrubbing: Comment on Power et al. Neuroimage 76: 436-438.

Carpenter WT Jr., Gold JM, Lahti AC, Queern CA, Conley RR, Bartko JJ et al (2000). Decisional capacity for informed consent in schizophrenia research. Arch Gen Psychiatry 57: 533-538.

Chumbley JR, Friston KJ (2009). False discovery rate revisited: FDR and topological inference using Gaussian random fields. Neuroimage 44: 62-70.

Clinton SM, Meador-Woodruff JH (2004). Thalamic dysfunction in schizophrenia: neurochemical, neuropathological, and in vivo imaging abnormalities. Schizophrenia Res 69: 237-253.

Cole DM, Beckmann CF, Searle GE, Plisson C, Tziortzi AC, Nichols TE et al (2012a). Orbitofrontal connectivity with resting-state networks is associated with midbrain dopamine D3 receptor availability. Cereb Cortex 22: 2784-2793.

Cole DM, Oei NY, Soeter RP, Both S, van Gerven JM, Rombouts SA et al (2012b). Dopamine-dependent architecture of corticosubcortical network connectivity. Cereb Cortex 23: 1509-1516.

Csernansky JG, Schindler MK, Splinter NR, Wang L, Gado M, Selemon LD et al (2004). Abnormalities of thalamic volume and shape in schizophrenia. Am J Psychiatry 161: 896-902.

Damoiseaux JS, Greicius MD (2009). Greater than the sum of its parts: a review of studies combining structural connectivity and resting-state functional connectivity. Brain Struct Funct 213: $525-533$.

Damoiseaux JS, Rombouts SA, Barkhof F, Scheltens P, Stam CJ, Smith SM et al (2006). Consistent resting-state networks across healthy subjects. Proc Natl Acad Sci USA 103: 13848-13853.

Del Arco A, Mora F (2009). Neurotransmitters and prefrontal cortex-limbic system interactions: implications for plasticity and psychiatric disorders. J Neural Transm 116: 941-952.

Fox MD, Raichle ME (2007). Spontaneous fluctuations in brain activity observed with functional magnetic resonance imaging. Nat Rev Neurosci 8: 700-711.

Fox MD, Snyder AZ, Vincent JL, Corbetta M, Van Essen DC, Raichle ME (2005). The human brain is intrinsically organized into dynamic, anticorrelated functional networks. Proc Natl Acad Sci UStates A 102: 9673-9678.

Gaspar P, Berger B, Febvret A, Vigny A, Henry JP (1989). Catecholamine innervation of the human cerebral cortex as revealed by comparative immunohistochemistry of tyrosine hydroxylase and dopamine-beta-hydroxylase. I Comparative Neurol 279: 249-271.

Goto Y, Grace AA (2007). The dopamine system and the pathophysiology of schizophrenia: a basic science perspective. Int Rev Neurobiol 78: 41-68.

Gu H, Salmeron BJ, Ross TJ, Geng X, Zhan W, Stein EA et al (2010). Mesocorticolimbic circuits are impaired in chronic cocaine users as demonstrated by resting-state functional connectivity. Neuroimage 53: 593-601.

Harrow M, Sands JR, Silverstein ML, Goldberg JF (1997). Course and outcome for schizophrenia versus other psychotic patients: a longitudinal study. Schizophr Bull 23: 287-303.
Holcomb HH, Cascella NG, Thaker GK, Medoff DR, Dannals RF, Tamminga CA (1996). Functional sites of neuroleptic drug action in the human brain: PET/FDG studies with and without haloperidol. Am J Psychiatry 153: 41-49.

Kapur S (2003). Psychosis as a state of aberrant salience: a framework linking biology, phenomenology, and pharmacology in schizophrenia. Am J Psychiatry 160: 13-23.

Kraguljac NV, Reid M, White D, Jones R, den Hollander J, Lowman $\mathrm{D}$ et al (2012). Neurometabolites in schizophrenia and bipolar disorder-A systematic review and meta-analysis. Psychiatry Res 203: $111-125$.

Lahti AC, Weiler MA, Medoff DR, Tamminga CA, Holcomb $\mathrm{HH}$ (2005). Functional effects of single dose first- and secondgeneration antipsychotic administration in subjects with schizophrenia. Psychiatry Res 139: 19-30.

Laruelle M, Abi-Dargham A, van Dyck CH, Gil R, D'Souza CD, Erdos J et al (1996). Single photon emission computerized tomography imaging of amphetamine-induced dopamine release in drug-free schizophrenic subjects. Proc Natl Acad Sci USA 93: 9235-9240.

Liddle PF, Lane CJ, Ngan ET (2000). Immediate effects of risperidone on cortico-striato-thalamic loops and the hippocampus. Br J Psychiatry 177: 402-407.

Lieberman JA, Stroup TS, McEvoy JP, Swartz MS, Rosenheck RA, Perkins DO et al (2005). Effectiveness of antipsychotic drugs in patients with chronic schizophrenia. $N$ Engl J Med 353: 1209-1223.

Lui S, Deng W, Huang X, Jiang L, Ma X, Chen H et al (2009). Association of cerebral deficits with clinical symptoms in antipsychotic-naive first-episode schizophrenia: an optimized voxel-based morphometry and resting state functional connectivity study. Am J Psychiatry 166: 196-205.

Lui S, Li T, Deng W, Jiang L, Wu Q, Tang H et al (2010). Shortterm effects of antipsychotic treatment on cerebral function in drug-naive first-episode schizophrenia revealed by 'resting state' functional magnetic resonance imaging. Arch Gen Psychiatry 67: 783-792.

Marder SR, Essock SM, Miller AL, Buchanan RW, Davis JM, Kane JM et al (2002). The Mount Sinai conference on the pharmacotherapy of schizophrenia. Schizophr Bull 28: 5-16.

Meador-Woodruff JH, Clinton SM, Beneyto M, McCullumsmith RE (2003). Molecular abnormalities of the glutamate synapse in the thalamus in schizophrenia. Ann N Y Acad Sci 1003: 75-93.

Nurnberger JI Jr., Blehar MC, Kaufmann CA, York-Cooler C, Simpson SG, Harkavy-Friedman J et al (1994). Diagnostic interview for genetic studies. Rationale, unique features, and training. NIMH Genetics Initiative. Arch Gen Psychiatry 51: 849-859 discussion 863-864.

Overall JE, Gorham DR (1962). The brief psychiatric rating scale. Psychological Rep 10: 799-812.

Perez-Costas E, Melendez-Ferro M, Roberts RC (2010). Basal ganglia pathology in schizophrenia: dopamine connections and anomalies. J Neurochem 113: 287-302.

Power JD, Barnes KA, Snyder AZ, Schlaggar BL, Petersen SE (2012a). Spurious but systematic correlations in functional connectivity MRI networks arise from subject motion. Neuroimage 59: 2142-2154.

Power JD, Barnes KA, Snyder AZ, Schlaggar BL, Petersen SE (2012b). Steps toward optimizing motion artifact removal in functional connectivity MRI; a reply to Carp. Neuroimage 76: 439-441.

Raichle ME, MacLeod AM, Snyder AZ, Powers WJ, Gusnard DA, Shulman GL (2001). A default mode of brain function. Proc Natl Acad Sci USA 98: 676-682.

Randolph C, Tierney MC, Mohr E, Chase TN (1998). The Repeatable Battery for the Assessment of Neuropsychological Status (RBANS): preliminary clinical validity. I Clin Exp Neuropsychol 20: 310-319. 
VTA functional connectivity in schizophrenia

JA Hadley et al

Salamone JD, Correa M (2012). The mysterious motivational functions of mesolimbic dopamine. Neuron 76: 470-485.

Sambataro F, Blasi G, Fazio L, Caforio G, Taurisano P, Romano R et al (2010). Treatment with olanzapine is associated with modulation of the default mode network in patients with Schizophrenia. Neuropsychopharmacology 35: 904-912.

Seeley WW, Menon V, Schatzberg AF, Keller J, Glover GH, Kenna $\mathrm{H}$ et al (2007). Dissociable intrinsic connectivity networks for salience processing and executive control. J Neurosci 27: 2349-2356.

Shepherd AM, Laurens KR, Matheson SL, Carr VJ, Green MJ (2012). Systematic meta-review and quality assessment of the structural brain alterations in schizophrenia. Neurosci Biobehav Rev 36: 1342-1356.

Skidmore FM, Yang M, Baxter L, von Deneen KM, Collingwood J, $\mathrm{He} \mathrm{G}$ et al (2013). Reliability analysis of the resting state can sensitively and specifically identify the presence of Parkinson disease. Neuroimage 75: 249-261.

Sodhi MS, Simmons M, McCullumsmith R, Haroutunian V, Meador-Woodruff JH (2011). Glutamatergic gene expression is specifically reduced in thalamocortical projecting relay neurons in schizophrenia. Biol Psychiatry 70: 646-654.

Song XW, Dong ZY, Long XY, Li SF, Zuo XN, Zhu CZ et al (2011). REST: a toolkit for resting-state functional magnetic resonance imaging data processing. PLoS ONE 6: e25031.

Tomasi D, Volkow ND (2012). Functional connectivity of substantia nigra and ventral tegmental area: maturation during adolescence and effects of ADHD. Cereb Cortex (e-pub ahead of print 12 December 2012; doi:10.1093/cercor/bhs382).

Whitfield-Gabrieli S, Ford JM (2012). Default mode network activity and connectivity in psychopathology. Annu Rev Clin Psychol 8: 49-76.

Supplementary Information accompanies the paper on the Neuropsychopharmacology website (http://www.nature.com/npp) 